\title{
Fatal pulmonary microthrombi during surgical therapy for end-stage heart failure: Possible association with antifibrinolytic therapy
}

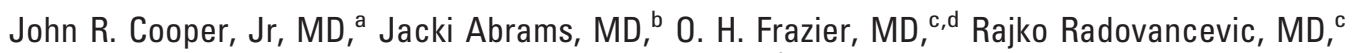

Branislav Radovancevic, MD, ${ }^{c}$ Arthur W. Bracey, MD, Michel J. Kindo, MD, ${ }^{c}$ and Igor D. Gregoric, MD ${ }^{\mathrm{c}, \mathrm{d}}$

From the Departments of a Cardiovascular Anesthesiology, ${ }^{\mathrm{b}}$ Pathology, ${ }^{\mathrm{c}}$ Cardiovascular Surgery, and Cardiovascular Surgical and Transplant Research, Texas Heart Institute at St. Luke's Episcopal Hospital, Houston, Tex.

Received for publication May 25, 2005; revisions received Nov 10, 2005; accepted for publication Jan 10, 2006.

Address for reprints: O. H. Frazier, MD, P.O. Box 20345, MC 3-147, Houston, TX 77225-0345 (E-mail: knowlin@ @eart.thi. tmc.edu).

J Thorac Cardiovasc Surg 2006;131:963-8

$0022-5223 / \$ 32.00$

Copyright $(\odot) 2006$ by The American Association for Thoracic Surgery

doi:10.1016/j.jtcvs.2006.01.005
Background: Maintaining hemostasis in patients with end-stage heart failure undergoing cardiac surgery is always challenging. These patients have chronic hepatic insufficiency, resulting in derangement of coagulation. In addition, they are commonly receiving both systemic anticoagulation (warfarin or heparin) and antiplatelet therapy. The introduction of antifibrinolytics has had a significant effect on postoperative coagulopathy. We report fatal pulmonary microthrombi in patients receiving antifibrinolytics who developed suprasystemic pulmonary artery pressures and right heart failure that was impossible to overcome despite insertion of a right ventricular assist device.

Methods: We reviewed the surgical procedure and autopsy reports to identify patients with high pulmonary artery pressures caused by pulmonary microthrombi after a cardiac surgical procedure for end-stage heart failure. Patient demographics and preoperative, intraoperative, and postoperative variables were collected from a retrospective review of the patients' medical records.

Results: We identified 9 patients ( 7 men and 2 women; mean age, $45 \pm 16$ years) who died of pulmonary microthrombi after cardiac surgery between January 1997 and January 2004. Surgical procedures included 5 left ventricular assist device implantations, 2 heart transplantations, and 2 left ventricular reconstructions with mitral valve repair or replacement. Eight patients received aprotinin, and 1 patient received $\varepsilon$-aminocaproic acid immediately before and during cardiopulmonary bypass. All patients had severe suprasystemic pulmonary artery pressures after protamine administration for heparin reversal, a complication that proved fatal in all cases. Intraoperative wedge biopsy of the lungs revealed multiple microthrombi within capillaries and in the small- and medium-sized pulmonary arterioles.

Conclusion: We report 9 cases for which fatal pulmonary microthrombi might be associated with the use of prophylactic antifibrinolytic therapy. Mortally ill patients with multiorgan failure who are receiving systemic anticoagulation and undergoing surgical procedures require careful perioperative monitoring to identify potential hazards. Anticoagulation and antifibrinolytic therapy protocols may require adjustment in such patients.

$\mathrm{S}$ urgical therapy for critically ill patients with heart failure is associated with a variety of complications. In 1997, one of the authors (O.H.F.) experienced a mortality in a high-risk patients after insertion of a left ventricular assist device (LVAD) who experienced suprasystemic pulmonary artery pressures and in whom right heart failure was impossible to overcome despite insertion of a right ventricular assist device (RVAD). A frozen section performed on an intraoperative wedge biopsy specimen of the lung showed, on close inspection, multiple recent microthrombi in the small vessels, a finding not previously identified in such 


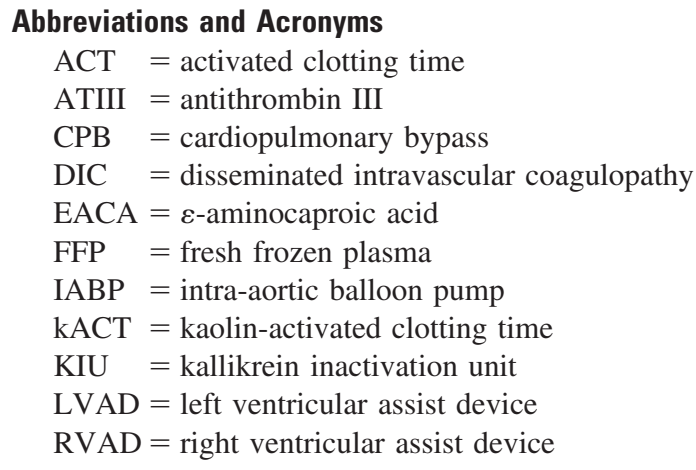

patients. During the next 7 years, we identified 8 additional such patients. In all of these patients, antifibrinolytic therapy was administered immediately before and throughout the cardiopulmonary bypass (CPB) period. Intraoperative frozen section and postmortem examination confirmed the presence of multiple microthrombi within the capillaries and small- and occasionally medium-sized pulmonary arterioles.

\section{Methods}

We reviewed our database, patient records, and autopsy reports to identify patients who had died either immediately or soon after undergoing surgical treatment for end-stage heart failure and who had associated pulmonary microthrombi with suprasystemic pulmonary artery pressure. Data collected included age, sex, weight, medical history, diagnosis, operative procedure and urgency, and duration of CPB. All drugs administered intraoperatively, results of intraoperative coagulation tests, operative complications (including bleeding), and all allogeneic blood transfusions were reviewed. Categoric variables are described by frequency (in percentages), and continuous measures are described as the mean \pm standard deviation.

\section{Results}

Patients

We identified 9 patients ( 7 men and 2 women; mean age, 45 \pm 16 years; range, 19-60 years) who died either during or immediately after cardiac surgery between January 1997 and January 2004 whose frozen section wedge lung biopsy specimens showed extensive microthrombi in the pulmonary vasculature. Patient demographics and procedural data are presented in Table 1.

All patients were referred for surgical treatment of severe end-stage heart failure (New York Heart Association functional class IV) caused by dilated cardiomyopathy. All 9 patients had undergone previous cardiac procedures, and of these patients, 5 had received preoperative circulatory support with an intra-aortic balloon pump (IABP). All patients had hepatic and renal dysfunction at the time of surgical intervention. All 9 patients had received long-term oral anticoagulation with warfarin in association with their heart failure treatment. Seven patients received intravenous heparin therapy. Neither the patients nor their blood relatives had any preexisting history of deep venous thrombosis, pulmonary emboli, or stroke preoperatively. Preoperative characteristics were not significant for allergic reactions to drugs. Routine coagulation monitoring was performed in all patients.

All 9 patients had advanced cardiac failure requiring pharmacologic support to maintain cardiac output. In addition, 7 of the 9 patients required preoperative IABP counterpulsation; 2 patients arrived in the operating room with cardiopulmonary resuscitation in progress (Table 2).

\section{Surgical Procedures}

Five of the 9 patients underwent LVAD implantation, 2 underwent heart transplantation, and the remaining 2 underwent left ventricular reconstruction with mitral valve repair or replacement (Table 1).

TABLE 1. Patient demographics and procedural data

\begin{tabular}{|c|c|c|c|c|c|c|}
\hline Patient no. & Sex & Age (y) & Weight (kg) & Procedure & $\begin{array}{l}\text { Initial duration of } \\
\text { CPB (min) }\end{array}$ & Antifibrinolytic \\
\hline 1 & $M$ & 53 & 66 & HT & 106 & Aprotinin (HDP) \\
\hline 2 & $\mathrm{M}$ & 42 & 71 & HT & 125 & Aprotinin (HDP) \\
\hline 3 & $\mathrm{M}$ & 19 & 68 & LVR + MVP & 167 & EACA \\
\hline 4 & $\mathrm{M}$ & 60 & 82 & LVAD & 74 & Aprotinin (HDP) \\
\hline 5 & $\mathrm{M}$ & 55 & 62 & LVR + MVR & 136 & Aprotinin (BWP) \\
\hline 6 & $\mathrm{~F}$ & 47 & 101 & LVAD & 177 & Aprotinin (HDP) \\
\hline 7 & $\mathrm{M}$ & 56 & 80 & LVAD & 90 & Aprotinin (BWP) \\
\hline 8 & $\mathrm{~F}$ & 56 & 70 & LVAD & 71 & Aprotinin (BWP) \\
\hline 9 & $\mathrm{M}$ & 20 & 100 & LVAD & 84 & Aprotinin (BWP) \\
\hline
\end{tabular}

$C P B$, Cardiopulmonary bypass; $H T$, heart transplantation; $H D P$, high-dose protocol; $L V R$, left ventricular reconstruction; $M V P$, mitral valvuloplasty; $E A C A$, $\varepsilon$-aminocaproic acid; $L V A D$, left ventricular assist device; $M V R$, mitral valve replacement; $B W P$, body-weight protocol. 
TABLE 2. Preoperative patient condition and intervention

\begin{tabular}{cllll}
\hline Patient no. & \multicolumn{1}{c}{ Diagnosis } & Planned procedure & \multicolumn{1}{c}{ Preoperative condition } & Preoperative intervention \\
\hline 1 & CMP (ISC) & HT & CHF, hypotensive & Inotropes \\
2 & CMP post-LVR & HT & CHF, renal dysfunction & Diuretics, antidiuretics, $\beta$-blockers \\
3 & CMP postcardiac trauma & LVR + MVP & Ventricular fibrillation & CPR in progress in OR, vasopressors \\
4 & CMP (ISC) post-LVR & LVAD & CHF, respiratory failure, renal failure & IABP, inotropes, vasopressors \\
5 & CMP & LVR +MVR & CHF & IABP, inotropes \\
6 & CMP post-HHT & LVAD & CHF & Inotropes \\
7 & CMP & LVAD & CHF, cardiac arrest, respiratory & CPR in progress in OR, IABP, \\
& CMP & LVAD & failure & vasopressors \\
& & & failure respiratory failure, hepatic & IABP, vasopressors
\end{tabular}

CMP, Cardiomyopathy; ISC, ischemic; $H T$, heart transplantation; $C H F$, congestive heart failure; $L V R$, left ventricular reconstruction; $M V P$, mitral valvuloplasty; $C P R$, cardiopulmonary resuscitation; $O R$, operating room; $L V A D$, left ventricular assist device; $I A B P$, intra-aortic balloon pump; $M V R$, mitral valve replacement; HHT, heterotopic heart transplant.

All procedures were performed with membrane oxygenators. The CPB circuit was primed either with electrolyte solution (5 patients) or fresh frozen plasma (FFP; 4 patients). Packed red blood cells were added to the pump only if the patient's hemoglobin level was less than $6 \mathrm{~g} / \mathrm{dL}$ at $30^{\circ} \mathrm{C}$. Anesthesia induction was performed with etomidate or thiopental plus fentanyl $(5-10 \mathrm{mg} / \mathrm{kg})$. Low-dose isoflurane $(0.4 \%-1.0 \%)$ was used for anesthesia maintenance and was supplemented with midazolam $(5-10 \mathrm{mg})$ and fentanyl (5-10 $\mathrm{mg} / \mathrm{kg})$. Anticoagulation was achieved with heparin ( $3 \mathrm{mg} / \mathrm{kg}$ or $300 \mathrm{U} / \mathrm{kg}$ body weight). Additional heparin was administered to maintain the kaolin-activated clotting time (kACT) at longer than 450 to 500 seconds. At the end of the CPB period, intravenous protamine sulfate was administered for heparin reversal. Two patients were systemically cooled by means of CPB (approximately $28^{\circ} \mathrm{C}$ ), and the others underwent the procedure under hypothermic $\left(34^{\circ} \mathrm{C}\right)$ conditions. All patients were rewarmed to a nasopharyngeal temperature of $36.5^{\circ} \mathrm{C}$ before being weaned from CPB. Median initial CPB time was 114 minutes (range, 71-177 minutes). After discontinuation of $\mathrm{CPB}$, there was no gross evidence of thrombus in any perfusion circuit.
In an effort to decrease postoperative bleeding, 8 patients received aprotinin, and 1 patient received $\varepsilon$-aminocaproic acid (EACA) (25-g total dose). Of the patients who received aprotinin, 4 received the high-dose aprotinin protocol: a 1-mL (10,000 kallikrein inactivation unit [KIU]) test dose, a 200-mL (2.0 million KIU) loading dose, and a $50 \mathrm{~mL} / \mathrm{h}$ $(500,000 \mathrm{KIU} / \mathrm{h})$ continuous infusion. On initiation of CPB, an additional $200 \mathrm{~mL}$ (2.0 million KIU) was added to the $\mathrm{CPB}$ priming fluid. In the other 4 patients, aprotinin was administered according to a weight-based protocol; the loading dose and CPB priming doses were $100 \mathrm{~mL}$ each. Three of the 8 patients who received aprotinin had prior exposure to aprotinin $(3,5$, and 32 days) before the current operation.

Average blood use was $15 \pm 10$ units of red blood cells, $17 \pm 17$ units of FFP, and $18 \pm 13$ units of platelets. (One patient was a Jehovah's Witness.) Cell saver was used in all patients. Salvaged blood was washed and returned to the patients.

Continuous monitoring of heparinization with kACT was performed in all patients. Additional heparin was added when needed to keep the activated clotting time

TABLE 3. ACT monitoring and heparin infusions during CPB

\begin{tabular}{cllr}
\hline Patient & Antifibrinolytic & \multicolumn{1}{c}{ kACT (s) } & Heparin (mg) \\
\hline 1 & Aprotinin & $624-430-507$ & 210 \\
2 & Aprotinin & $415 / 400-711-836-724-604-636$ & $210+50+50$ \\
3 & EACA & $480-406-400-294-403-521$ & 200 \\
4 & Aprotinin & $484-463-433-452-338$ & 250 \\
5 & Aprotinin & $362-375-419-400-497-474-437-425$ & $190+50+50$ \\
6 & Aprotinin & $999-536-587-716-587-758-734-656-733-700-631$ & $300-50-50$ \\
7 & Aprotinin & $740-387-443-553-608-576-666-909$ & $300+10+10+50+100+50+50+50+50$ \\
8 & Aprotinin & $999-999-999-999-999-999$ & $270+270$ \\
9 & Aprotinin & $571-655-660-494-306$ & $300+100+100$
\end{tabular}

$A C T$, Activated clotting time; $C P B$, cardiopulmonary bypass; $k A C T$, kaolin-activated clotting time; $E A C A$, $\varepsilon$-aminocaproic acid. 

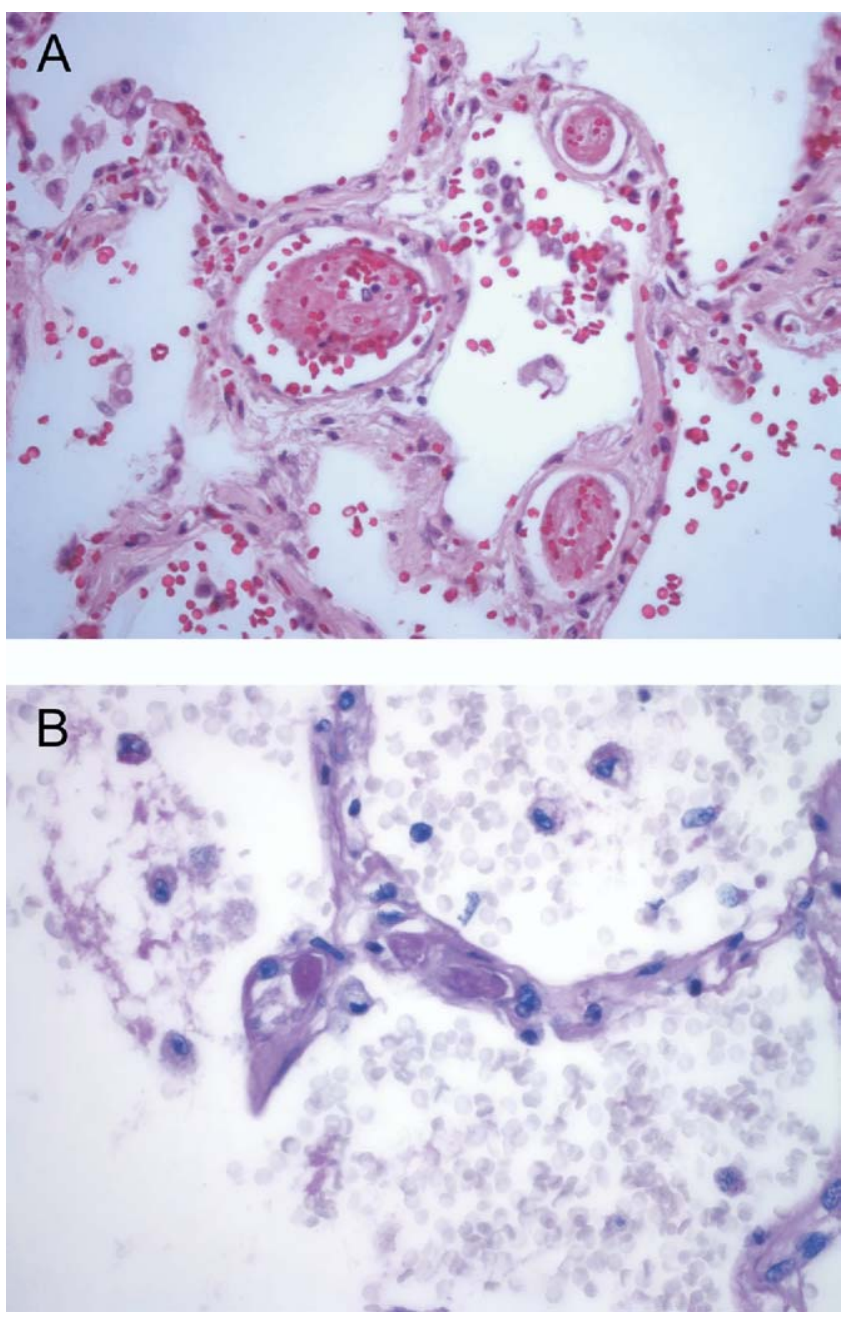

Figure 1. Three adjacent pulmonary capillaries occluded by fibrin thrombi ( $A$, hematoxylin and eosin stain, original magnification $400 \times$; B, periodic acid-Schiff stain, original magnification $600 \times$ ).

(ACT) in the target range, but there was some variation in the ACT value (in 6 of the 9 patients, the kACT was $<450$ seconds for short periods). In addition, the timing of the measurement of the kACT was inconsistent (Table 3). After completion of the procedure and decannulation, heparinization was reversed with protamine.

In all patients, within minutes of protamine administration, pulmonary artery pressure dramatically increased. In 1 patient after RVAD placement, a direct pressure measurement was greater than $300 \mathrm{~mm} \mathrm{Hg}$ (the limit of pressure that could be generated by the device). In all patients the pulmonary artery pressure was suprasystemic. Right ventricular distension and lack of right ventricular wall motion was observed. All patients again received heparin, full CPB was reinitiated, and RVAD implantation was performed. Despite RVAD support, 8 of the 9 patients died in the operating room. One patient received fibrinolytic therapy (tissue-type plasminogen activator), which reversed the pulmonary hypertension, but the patient died of exsanguination in the intensive care unit.

\section{Pathologic Findings}

Postmortem examinations were limited to the heart and lungs in 7 patients, and complete autopsies were performed in 2 patients. In all patients the histology was identical. Microscopically, there were multiple recent thrombi, which appeared to be composed of fibrin (with no obvious cellular component) present in the capillaries and small- and medium-sized pulmonary arterioles throughout both lungs (Figure 1). Three patients also were noted to have microthrombi present within the epicardial and intramyocardial microvasculature. In the 2 patients in whom full autopsies were performed, microthrombi were not noted in other organs.

\section{Discussion}

Fibrin microthrombi are a localized expression of a systemic process, such as disseminated intravascular coagulopathy (DIC). They have been found in various organs, including the lungs, in organ rejection episodes and in a variety of disease entities, such as DIC or hemolytic anemia syndrome among others, and are not thought to be an embolic phenomenon.

The clinical presentation of the patients in this series does not, in our view, reflect an embolic cause. All patients had been successfully weaned from CPB (with some inotropic support and, for some, IABP support) and had had their venous cannulas removed. Only after protamine reversal of heparin did the catastrophic pulmonary hypertension and subsequent right ventricular failure result. The fact that RVAD use was not able to overcome the increased pulmonary resistance, coupled with the uniform lack of response to pharmacologic therapy for pulmonary hypertension, speaks to the extent of the microthrombi occlusion of pulmonary arterioles and capillaries.

The overriding clinical presentation characterized by complete blockage of meaningful pulmonary blood flow has not been experienced at our institution and has no clinical compatibility with the hypotensive response occasionally seen with protamine administration. This dramatic cessation of pulmonary blood flow is unique in the experience of the senior author (O.H.F.), who was present during all of the cases in this report and has performed more than 500 LVAD implantations and more than 900 heart transplantation procedures.

In general, cardiac surgery is associated with an initial hypocoagulable state, although hypercoagulability might manifest later in the postoperative period. Many factors contribute to intraoperative and postoperative bleeding, including heparin use, platelet dysfunction, protein denaturation by 
means of CPB, hemodilution, hypothermia, inflammatory mediator release, and hyperfibrinolysis. Preoperative factors, such as liver and renal dysfunction and anticoagulant use, might also contribute to postoperative bleeding.

In an effort to combat bleeding after $\mathrm{CPB}$, pharmacologic therapy has been used as an adjunct to other measures, in particular to address hyperfibrinolysis. Antifibrinolytic therapy reduces hyperfibrinolysis and mitigates inflammatory disturbances that might contribute to coagulopathy. ${ }^{1}$ The perioperative use of antifibrinolytic drugs, such as aprotinin or EACA, reduces the incidence of blood loss, allogeneic blood transfusion, mediastinal re-exploration, and mortality after cardiac surgery. ${ }^{2-6}$

In all patients described in this report, antifibrinolytic therapy was administered. Several reports exist in the literature about thrombotic complications associated with the use of antifibrinolytics. Heindel and colleagues ${ }^{7}$ report a case of fatal systemic thrombosis associated with aprotinin use and heparin-protamine reversal during a hemi-Fontan procedure. Other investigators report an increased tendency toward thrombosis-related complications associated with aprotinin, including myocardial infarction, ${ }^{8}$ early saphenous vein graft thrombosis, ${ }^{9-11}$ thrombi formation on the pulmonary artery catheter after $\mathrm{CPB},{ }^{12}$ and fatal pulmonary embolism during liver transplantation. ${ }^{13-15}$ Another report of a massive intravascular thrombosis after cardiac arrest in a patient treated with EACA also raises concern about the agents used for antifibrinolytic therapy. ${ }^{16}$ However, in a meta-analysis by Levi and associates, ${ }^{2}$ the use of aprotinin during cardiac surgery was not associated with an increased risk of perioperative myocardial infarction. Furthermore, it has been demonstrated that aprotinin normally seems to be simultaneously hemostatic and antithrombotic because it selectively blocks the proteolytically activated thrombin receptor on platelets but leaves other mechanisms of platelet aggregation unaffected. ${ }^{17}$

Further controversy revolves around the amount of antifibrinolytic agent that should be used, especially in the case of aprotinin. High-dose, low-dose, and weight-based protocols have been advocated. Four of the patients in this series received the high-dose protocol, and 4 received a weightbased protocol, challenging the association of the amount of aprotinin administered as being a significant factor in the clinical course of these patients.

Anticoagulation management during $\mathrm{CPB}$ is controversial, especially when using a potentially unreliable test, such as ACT, in the presence of aprotinin. ${ }^{18,19}$ Current recommendations suggest that heparin be administered with the goal of a celite ACT of greater than 750 seconds $^{20}$ and a kACT of greater than 480 seconds, although these values are arbitrary and controversial. ${ }^{21}$ EACA does not interfere with the kACT measurement in the presence of heparin. ${ }^{22}$ In our patients additional heparin was used to maintain the
kACT at greater than 480 seconds; however, in some cases there were short periods when the kACT did not reach this level. In others the kACT values were consistently high, which, by conventional standards, indicates that heparinization was adequate.

Most of the patients in the case reports or series cited earlier had gross systemic or venous thrombosis, which is a clinically different presentation than we observed. However, because we were clearly unable to achieve transpulmonary blood flow, it is not surprising that the microthrombi would be limited to the lungs. Sundt and coworkers ${ }^{23}$ report the presence of platelet-fibrin thrombi in a series of patients in whom aprotinin and hypothermic circulatory arrest were used. In that study the platelet-fibrin thrombi were found in multiple organs, but in 2 patients there was significant pulmonary involvement. Of these patients, 1 died intraoperatively of acute right ventricular failure. Like Sundt and coworkers, we have not observed this complication in any of our patients of this type in whom aprotinin or EACA was not used. In addition, we have only observed it in 9 patients. We do not routinely use antifibrinolytics in all critically ill patients, and therefore the denominator population for this report is unknown; however, we do routinely prime the $\mathrm{CPB}$ circuit with FFP and avoid use of electrolyte solutions in patients with advanced heart failure.

Although antifibrinolytic therapy might be a factor in this syndrome, clearly others must also be involved. First, all patients in this group had poor cardiac function (resulting in low cardiac output), liver and renal dysfunction, and poor peripheral perfusion. Although liver and renal dysfunction generally promote hypocoagulability through a variety of mechanisms, they also might promote hypercoagulation through decreased production of endogenous anticoagulant factors and decreased clearance of activated procoagulants, producing DIC. However, it does not usually present with such a catastrophic effect.

Second, those endogenous anticoagulant factors might have been affected by other interventions. Preoperative heparin treatment induces decreased antithrombin III (ATIII) activity, which might result in a reduced sensitivity to heparin during CPB. ${ }^{24,25}$ In addition, hemodilution and ATIII consumption induced by $\mathrm{CPB}$ could cause ATIII levels to further decrease. Even in the presence of high heparin concentrations, the monitoring and substitution of ATIII appears to ensure more efficient antithrombin activity during $\mathrm{CPB} .{ }^{26}$ Although ATIII levels were not routinely measured in our patients, multiple FFP transfusions were given, and the CPB circuit was primed with FFP in 4 patients, which argues against ATIII depletion as a contributing factor.

Other endogenous anticoagulant factors, such as proteins $\mathrm{C}$ and $\mathrm{S}$, when deficient, can be associated with hypercoagulation. Pathologic states, such as the antiphospholipid 
syndrome or the presence of Factor V Leiden, might also be associated with hypercoagulability; unfortunately, their presence or absence was not measured in our patients, and therefore their effect is unknown.

Although this report is limited by its retrospective and observational nature, we believe these patients represent a unique syndrome. Therefore we believe it is important to report these observations.

\section{Conclusions}

The value of antifibrinolytic therapy in reducing blood loss has clearly been demonstrated. To safely extend its use in these critically ill patients, however, requires careful perioperative monitoring to identify any potential hazards, so that anticoagulation and antifibrinolytic therapy protocols can be adjusted accordingly. Particular attention should be paid to adequate heparinization throughout the sometimes prolonged CPB times required for performing complicated procedures in patients with advanced heart failure. Consideration should be given to continuous heparin infusion during CPB to avoid even short periods of undetected inadequate heparinization. In most patients extensive bleeding can be dealt with successfully, but extensive thrombosis was fatal in these patients.

\section{References}

1. Despotis GJ, Avidan MS, Hogue CW Jr. Mechanisms and attenuation of hemostatic activation during extracorporeal circulation. Ann Thorac Surg. 2001;72(suppl):S1821-31

2. Levi M, Cromheecke ME, de Jonge E, Prins MH, de Mol BJ, Briet E, et al. Pharmacological strategies to decrease excessive blood loss in cardiac surgery: a meta-analysis of clinically relevant endpoints. Lancet. 1999;354:1940-7.

3. Goldstein DJ, Seldomridge JA, Chen JM, Catanese KA, DeRosa CM, Weinberg $\mathrm{AD}$, et al. Use of aprotinin in LVAD recipients reduces blood loss, blood use, and perioperative mortality. Ann Thorac Surg. 1995;59:1063-7.

4. Goldstein DJ, Oz MC, Smith CR, Friedlander JP, De Rosa CM, Mongero LB, et al. Safety of repeat aprotinin administration for LVAD recipients undergoing cardiac transplantation. Ann Thorac Surg. 1996; 61:692-5

5. Smith CR. Management of bleeding complications in redo cardiac operations. Ann Thorac Surg. 1998;65(suppl):S2-8.

6. Levy JH. Pharmacologic preservation of the hemostatic system during cardiac surgery. Ann Thorac Surg. 2001;72(suppl):S1814-20.

7. Heindel SW, Mill MR, Freid EB, Valley RD, White GC 2nd, Norfleet EA. Fatal thrombosis associated with a hemi-Fontan procedure, heparin-protamine reversal, and aprotinin. Anesthesiology. 2001;94: 369-71.

8. Lemmer JH Jr, Dilling EW, Morton JR, Rich JB, Robicsek F, Bricker DL, et al. Aprotinin for primary coronary artery bypass grafting: a multicenter trial of three dose regimens. Ann Thorac Surg. 1996;62: 1659-67.
9. Lemmer JH Jr, Stanford W, Bonney SL, Breen JF, Chomka EV, Eldredge WJ, et al. Aprotinin for coronary bypass operations: efficacy, safety, and influence on early saphenous vein graft patency. A multicenter, randomized, double-blind, placebo-controlled study. J Thorac Cardiovasc Surg. 1994;107:543-51.

10. Alderman EL, Levy JH, Rich JB, Nili M, Vidne B, Schaff H, et al. Analyses of coronary graft patency after aprotinin use: results from the International Multicenter Aprotinin Graft Patency Experience (IMAGE) trial. J Thorac Cardiovasc Surg. 1998;116:716-30.

11. van der Meer J, Hillege HL, Ascoop CA, Dunselman PH, Mulder BJ, van Ommen GV, et al. Aprotinin in aortocoronary bypass surgery: increased risk of vein-graft occlusion and myocardial infarction? Supportive evidence from a retrospective study. Thromb Haemost. 1996; 75:1-3.

12. Bohrer H, Fleischer F, Lang J, Vahl C. Early formation of thrombi on pulmonary artery catheters in cardiac surgical patients receiving highdose aprotinin. J Cardiothorac Anesth. 1990;4:222-5.

13. Sopher M, Braunfeld M, Shackleton C, Busuttil RW, Sangwan S, Csete M. Fatal pulmonary embolism during liver transplantation. Anesthesiology. 1997;87:429-32.

14. Fitzsimons MG, Peterfreund RA, Raines DE. Aprotinin administration and pulmonary thromboembolism during orthotopic liver transplantation: report of two cases. Anesth Analg. 2001;92:1418-21.

15. Feindt P, Seyfert U, Volkmer I, Huwer H, Kalweit G, Gams E. Is there a phase of hypercoagulability when aprotinin is used in cardiac surgery? Eur J Cardiothorac Surg. 1994;8:308-13.

16. Haddy SM, Shely WW, Rice N. Intravascular thrombosis after exsanguination in a patient treated with epsilon-aminocaproic acid. $J$ Cardiothorac Vasc Anesth. 1996;10:510-2.

17. Landis RC, Asimakopoulos G, Poullis M, Haskard DO, Taylor KM. The antithrombotic and antiinflammatory mechanisms of action of aprotinin. Ann Thorac Surg. 2001;72:2169-75.

18. Despotis GJ, Gravlee G, Filos K, Levy J. Anticoagulation monitoring during cardiac surgery: a review of current and emerging techniques. Anesthesiology. 1999;91:1122-51.

19. Feindt P, Seyfert UT, Volkmer I, Straub U, Gams E. Celite and kaolin produce differing activated clotting times during cardiopulmonary bypass under aprotinin therapy. Thorac Cardiovasc Surg. 1994;42: 218-21.

20. Hunt BJ, Segal H, Yacoub M. Aprotinin and heparin monitoring during cardiopulmonary bypass. Circulation. 1992;86(suppl):II410-2.

21. Peters DC, Noble S. Aprotinin: an update of its pharmacology and therapeutic use in open heart surgery and coronary artery bypass surgery. Drugs. 1999;57:233-60.

22. Munoz JJ, Birkmeyer NJ, Birkmeyer JD, O'Connor GT, Dacey LJ. Is epsilon-aminocaproic acid as effective as aprotinin in reducing bleeding with cardiac surgery?: a meta-analysis. Circulation. 1999;99:81-9.

23. Sundt TM III, Kouchoukos NT, Saffitz JE, Murphy SF, Wareing TH, Stahl DJ. Renal dysfunction and intravascular coagulation with aprotinin and hypothermic circulatory arrest. Ann Thorac Surg. 1993;55: 1418-24.

24. Staples MH, Dunton RF, Karlson KJ, Leonardi HK, Berger RL. Heparin resistance after preoperative heparin therapy or intraaortic balloon pumping. Ann Thorac Surg. 1994;57:1211-6.

25. Dietrich W, Spannagl M, Schramm W, Vogt W, Barankay A, Richter JA. The influence of preoperative anticoagulation on heparin response during cardiopulmonary bypass. J Thorac Cardiovasc Surg. 1991;102: 505-14.

26. Koster A, Fischer T, Praus M, Haberzettl H, Kuebler WM, Hetzer R, et al. Hemostatic activation and inflammatory response during cardiopulmonary bypass: impact of heparin management. Anesthesiology. 2002;97:837-41. 